\title{
Origin and control of kinetic and thermodynamic hysteresis in clustering of gold nanoparticles
}

Joscha Kruse, ${ }^{\dagger}$ Stefan Merkens, ${ }^{\ddagger}$ Andrey Chuvilin, ${ }^{\ddagger}$ and Marek Grzelczak ${ }^{*, \dagger}$

†Centro de Física de Materiales CSIC-UPV/EHU and Donostia International Physics

Center (DIPC), Paseo Manuel de Lardizabal 5, 20018 Donostia-Sebastián, Spain $\ddagger$ ¿IC nanoGUNE BRTA, Tolosa Hiribidea 76, 20018 Donostia-Sebastián, Spain

【Ikerbasque, Basque Foundation for Science, 48013 Bilbao, Spain

E-mail: marek.g@csic.es 


\begin{abstract}
Hysteresis is an essential attribute of many solid-state devices and biological processes, yet it is often overlooked in colloidal and soft-matter dynamic systems. Herein we show that gold nanoparticles can remain dispersed or aggregated at the same temperature depending on the trajectory of applied stimulus, featuring hysteretic behaviour. Aided by real-time analytics and fine tuning of experimental parameters, such as salt concentration, nanoparticle diameter and surface potential, we disentangled kinetic (rate-dependent) and thermodynamic (rate-independent) component of hysteresis in cyclic clustering of nanoparticles. The hysteresis originates from the difference in the aggregation and disassembly temperatures. Our findings enrich the repertoire of experimental framework suitable for study life-like features on purely synthetic settings.
\end{abstract}

\title{
Graphical TOC Entry
}

\section{Keywords}

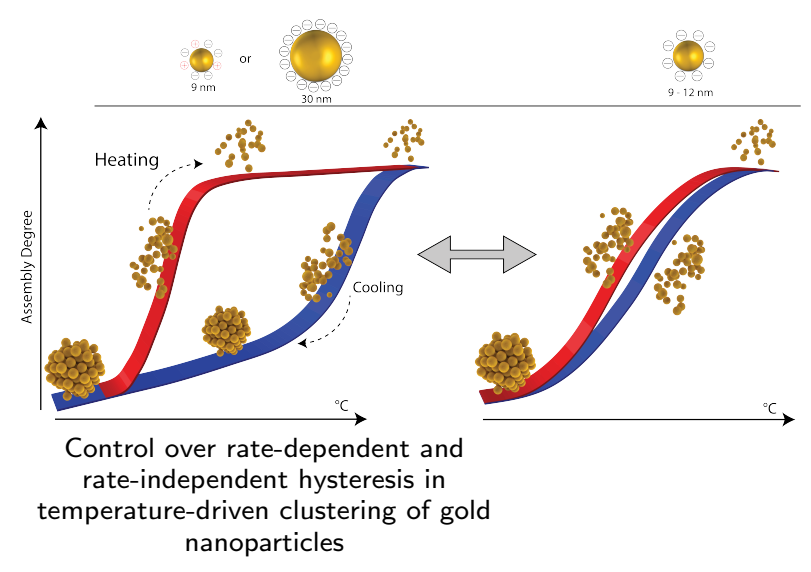

Reversible clustering, rate-dependent hysteresis, rate-independent hysteresis, gold nanoparticles, agarose, self-assembly 


\section{Introduction}

Apart from being a core feature in solid-state devices (e.g., memory storage), hysteresis sustains the functioning of many biological processes (e.g., cell division) through the resilience against noise. ${ }^{1-5}$ The studies of hysteresis require the use of suitable model systems. Without any doubt, one of such systems are gold nanoparticles (AuNPs) that exhibit chemical robustness under harsh conditions and optical properties sensitive to the change of their colloidal phase. ${ }^{6-11}$ Previously, Grzybowski and co-workers have studied hysteresis in the reversible clustering of gold nanoparticles stabilized with ionizable ligands using $\mathrm{pH}$ as a stimulus. ${ }^{12}$ These authors suggested that the hysteresis emerges from the change of magnitude of electrostatic repulsion that overcomes the energy barrier during the cyclic transitions between aggregated and dispersed states. The redispersion of nanoparticles required an extra change of $\mathrm{pH}$ to bring the nanoparticles out from the energy minimum. Importantly, the nanoparticles remained stable for several months once in aggregated state, indicating a local equilibrium adapted by the system over an entire range of applied stimulus. In this type of systems, the change of state is independent of the rate of applied stimulus, and the emerging hysteresis is classified as rate-independent.

An entirely different scenario represents rate-dependent hysteresis that originates from a lag of the system response to a stimulus. The faster is the velocity of the applied stimulus, the wider becomes the hysteresis; it is a kinetic effect. Rate-dependent hysteresis has been observed in pH-driven clustering of AuNPs stabilized with carboxylated alkanethiols, ${ }^{13-16}$ poly-L-lysine, ${ }^{17}$ and thermoresponsive polymers demonstrating an upper ${ }^{18}$ or lower ${ }^{19}$ critical solution temperature. Despite a vast body of works devoted to dynamic self-assembly, ${ }^{20-25}$ the hysteresis, when observed, receives mainly a qualitative description, needless to mention the lack of experimental control over its rate-dependent or rate-independent components. A reason for this is an insufficient time-resolution of data acquisition (e.g., spectroscopy) that could otherwise aid information and understanding of intermediate states in such systems. The knowledge of the type of hysteresis is central to predicting how the system responds to 
a stimulus. Rate-dependent hysteresis helps to describe the nonlinear relationship between the input stimulus and the output state, as in piezoelectric actuators. ${ }^{26}$ Rate-independent hysteresis, on the other hand, is a principle of memory storage, homeostatic materials, ${ }^{27}$ autonomous chemical reactions ${ }^{28}$ and erasable inks. ${ }^{29}$ Therefore, determining the signatures of the system response to a stimulus is a must in the development of functional nanosystems for (bio)sensors, actuators or catalysis.

The present work asks what is the origin of hysteretic behaviour during reversible clustering of nanoparticles and what are the experimental parameters that define the prevalence of either rate-dependent or rate-independent components. To answer this question, we selected temperature as a stimulus that modulates the electrostatic repulsion between nanoparticles. We observed the nanoparticles reside at different aggregation states at the same temperature depending on the direction of applied stimulus - cooling or heating. The ionic strength, which is modulated by salt and ligand concentration, defines the centre of hysteresis within a temperature window of $5-45^{\circ} \mathrm{C}$. The rate of heating or cooling determines the width of hysteresis, indicating rate-dependent component. Aided by real-time UV-Vis spectroscopy, we observed that the system is unable to relax equally from both aggregated and dispersed states at a given temperature to reach the state of minimum global energy, featuring rateindependent hysteresis. It can be further modulated by the diameter and surface potential of nanoparticles. Overall, our observations and presented data analysis offer a new tool in formulating the principles of hysteresis in dynamic chemical systems.

\section{Results and discussion}

We used citrate-stabilized AuNPs of $12 \pm 0.8 \mathrm{~nm}$ in diameter, that were functionalized with bis(p-sulfonatophenyl)-phenylphosphine (BSPP). ${ }^{30}$ Upon a temperature drop from 45

to $5{ }^{\circ} \mathrm{C}$, the initial red colour of the samples progressively turned to blue-grey (Figure 1a) and recovered by heating up to $45^{\circ} \mathrm{C}$. We recorded simultaneously the UV-Vis-NIR spectra 
and temperature values of the samples every 10 seconds (1000 spectra per run), observing that the localized surface plasmon band became featureless at $5{ }^{\circ} \mathrm{C}$ and recovered upon heating (Figure 1b). By excluding time component and presenting a degree of assembly $\left(R=A b s_{650 \mathrm{~nm}} / A b s_{520 \mathrm{~nm}}\right)$ versus temperature, we observed that $R$ follows different pathways depending on whether the sample is being cooled or heated, presenting hysteresis (Figure $1 \mathrm{c}, \mathrm{d})$.
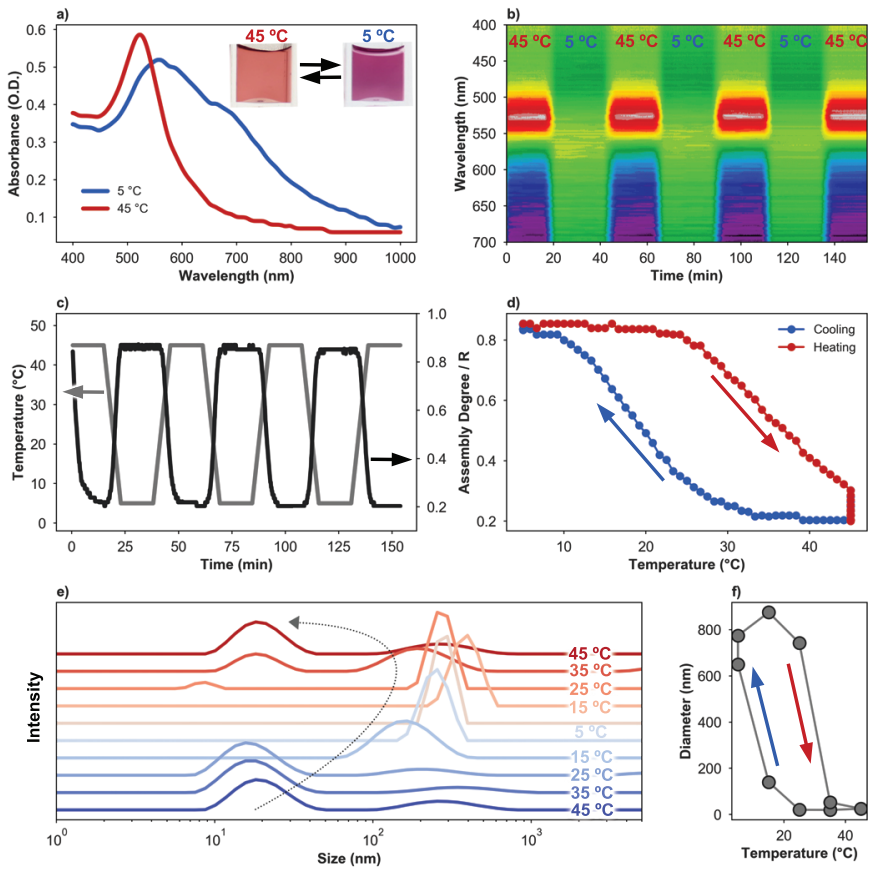

Figure 1: Temperature-driven reversible clustering of gold nanoparticles. a) UV-Vis-NIR spectra of redispersed (red, $45^{\circ} \mathrm{C}$ ) and aggregated (blue, $5^{\circ} \mathrm{C}$ ) nanoparticles. b) Four cycles of nanoparticles clustering at low and high temperatures. c) Time-dependent variation of temperature and assembly degree, $R$. d) Hysteresis of cyclic aggregation obtained by removal of time component in plot c. e, f) Change of hydrodynamic diameter of nanoparticles in colloidal phase at different temperatures confirming hysteresis.

Dynamic light scattering measurements confirmed the presence of hysteresis (Figure 1e,f, and S1). The hydrodynamic diameter varied between 30 and $800 \mathrm{~nm}$ during heating and cooling, respectively (Figure 1e). Extended storage of the sample at $5^{\circ} \mathrm{C}$ caused gradual phase separation that was prevented by dispersing the nanoparticles in an agarose matrix of pore size of $\sim 600 \mathrm{~nm}$ in diameter (Figure S2). ${ }^{31}$ The optimal agarose concentration was found to be $0.25 \mathrm{wt} \%$ (Figure S3). 
To assess to what extent a temperature gradient influences the hysteresis, we aggregated the nanoparticles cyclically in cuvettes of 5,2 and $1 \mathrm{~mm}$ path lengths. With decreasing the cuvette thickness, decreased hysteresis width. Such a tendency stems from the design of our setup. The light beam passes through the sample vertically from the source to spectrometer while the heat/cool is supplied from the bottom. The temperature gradient across the vertical axis generates a cluster size distribution along that axis, broadening the shape of surface plasmon resonance (For a schematic representation of cluster gradient see Figure S4). In $1 \mathrm{~mm}$ cuvette, the effect of the temperature gradient is brought to a minimum, so is the width of hysteresis.

\section{Centring hysteresis within temperature window}

The concentration of both salts $\mathrm{NaCl}$ and BSPP regulates the centre of the hysteresis in the temperature window of $5-45^{\circ} \mathrm{C}$. Note that BSPP is also a surface ligand here that binds to gold surface via phosphine group and ensures negative surface charge. With increasing $\mathrm{NaCl}$ concentration from 0 to $128 \mathrm{mM}$ at room temperature, the red colour of the samples turned blue (Figure 2a). Cyclic cooling/heating each of these samples revealed that the change of assembly degree, $R$, is the highest for intermediate salt concentrations $(64,80$ and $90 \mathrm{mM}$ ) and negligible for limiting concentrations (16, $120 \mathrm{mM}$ ) (Figure 2b, d). Consecutively, the centre of the hysteresis moved towards high temperature as salt concentration increased. A minimum concentration of BSPP $(1.5 \mathrm{mM})$ was needed to observe reversible aggregation, suggesting an additional role of free ligand in clustering process (Figure 2c, Figure S5). Similar scenario has been observed for free polymer molecules in temperaturedriven aggregation of nanoparticles stabilized with poly(N-isopropylacrylamide). ${ }^{10,32}$ Here, the change of assembly degree received the highest values at BSPP concentration of $3 \mathrm{mM}$. As for $\mathrm{NaCl}$, the increase of BSPP concentration moved the centre of hysteresis towards higher temperature. BSPP, however, alters the hysteresis at a larger extent. We speculate that the surface charge of AuNPs is screened twice as much by BSPP as compared to NaCl, 

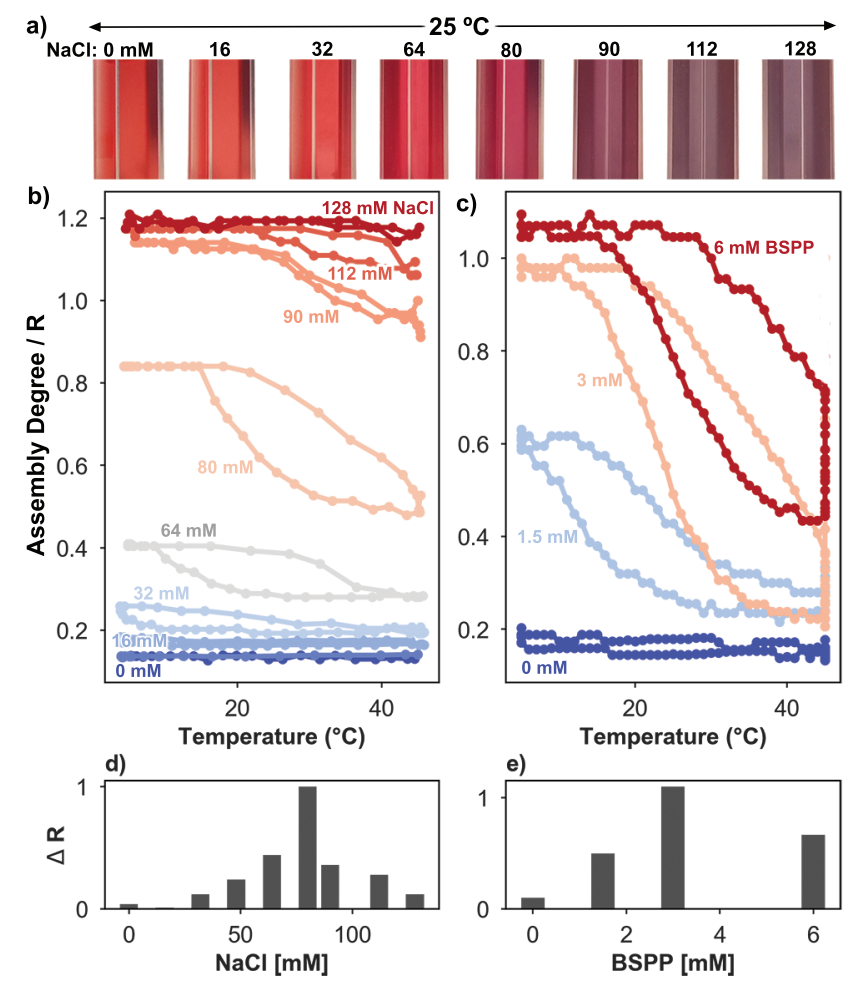

Figure 2: The effect of $\mathrm{NaCl}$ and BSPP concentration on the centre and saturation of hysteresis. a) Digital images of solutions containing gold nanoparticles in agarose matrix with increasing concentration of $\mathrm{NaCl}$ at $25^{\circ} \mathrm{C}$. b,c) With increasing the concentration of a) $\mathrm{NaCl}$ and b) BSPP the centre of hysteresis shifts to higher temperature at $1{ }^{\circ} \mathrm{C} / \mathrm{min}$. d, e) The change of responsiveness of the system $\left(\Delta R=R_{\max }-R_{\min }\right)$ to the concentration of $\mathrm{NaCl} \mathrm{d)}$ and BSPP e).

that is due to the presence of two potassium cations per one molecule of BSPP. Replacing BSPP with an alternative ligand - triphenylphosphine-3,3',3"-trisulfonic acid trisodium salt - had similar effect on the hysteresis (Figure S10).

To confirm the effect of temperature and salt concentrations on reversible aggregation, we calculated interaction potential between nanoparticles by using DLVO theory (Figures S6 and S7). The calculation showed that, indeed, the clustering is allowed in experimental temperature window $\left(5-45^{\circ} \mathrm{C}\right)$ for $\mathrm{NaCl}$ concentrations ranging from 64 to $80 \mathrm{mM}$ at a fixed amount of BSPP $(3 \mathrm{mM})$ and nanoparticles diameter of $12 \mathrm{~nm}$. Below the optimum $\mathrm{NaCl}$ concentrations the values of interaction potential are positive (dispersion), while above are negative (aggregation) regardless of temperature. Therefore, the numerical results confirm our experimental data. 
We studied the effect of particles concentration, observing that with increasing their concentration, the assembly degree increases since more particles are available to form clusters. As a result, the magnitude of hysteresis became larger (Figure S8). The centre of hysteresis, however, remained fixed at $20^{\circ} \mathrm{C}$ regardless of the particle concentration, so does the onset temperature in the cooling direction, at which the aggregation begins. Overall, in contrast to the concentration of particles, it is the concentration of $\mathrm{NaCl}$ and $\mathrm{BSPP}$ that influences the width and the centre of hysteresis within the temperature range, confirming the central role of electrostatic screening in reversible clustering.

\section{Disentangling rate-independent and rate-dependent hysteresis}

To confirm rate-dependent hysteresis, we performed cyclic clustering of the samples by cooling and heating for three velocities of temperature scan $\left(0.2,1\right.$ and $\left.5{ }^{\circ} \mathrm{C} / \mathrm{min}\right)$, observing progressive broadening of the hysteresis (Figure 3a). At the slowest scan rate, however, the hysteresis remained open (Figure 3a). To confirm whether it is an artefact or a sign of thermodynamic hysteresis, we performed a stair-like hysteresis where fast scans at 20 ${ }^{\circ} \mathrm{C} / \mathrm{min}$ (horizontal arrows in Figure 3b) was interrupted with a long relaxation period (20 min, vertical arrows in Figure $3 \mathrm{~b}$ ) at 15,25 , and $35^{\circ} \mathrm{C}$, allowing the system to relax at a given temperature during heating or cooling process. As a result, the assembly degree after relaxation at 25 and $35{ }^{\circ} \mathrm{C}$ coincided with assembly degree of the uninterrupted scan at 0.2 ${ }^{\circ} \mathrm{C} / \mathrm{min}$ (Figure 3i). The hysteresis thus contains rate-independent component.

A high temporal resolution of optical data acquisition allowed us to perform a qualitative analysis of the relaxation dynamics to targeted temperatures in stair-like hysteresis (Figure 3b). At the cooling or heating step, a fast change of temperature caused an abrupt variation of the assembly degree followed by its slow relaxation (Figure 3c). Pairwise comparison of relaxation profiles to a given temperature revealed the presence of a gap between assembly degrees of cooling and heating (Figure 3e-f). A fitting the relaxations at targeted temperatures $\left(45,35,25\right.$, and $\left.15{ }^{\circ} \mathrm{C}\right)$ revealed that on average, the aggregation takes $\sim 10$ 

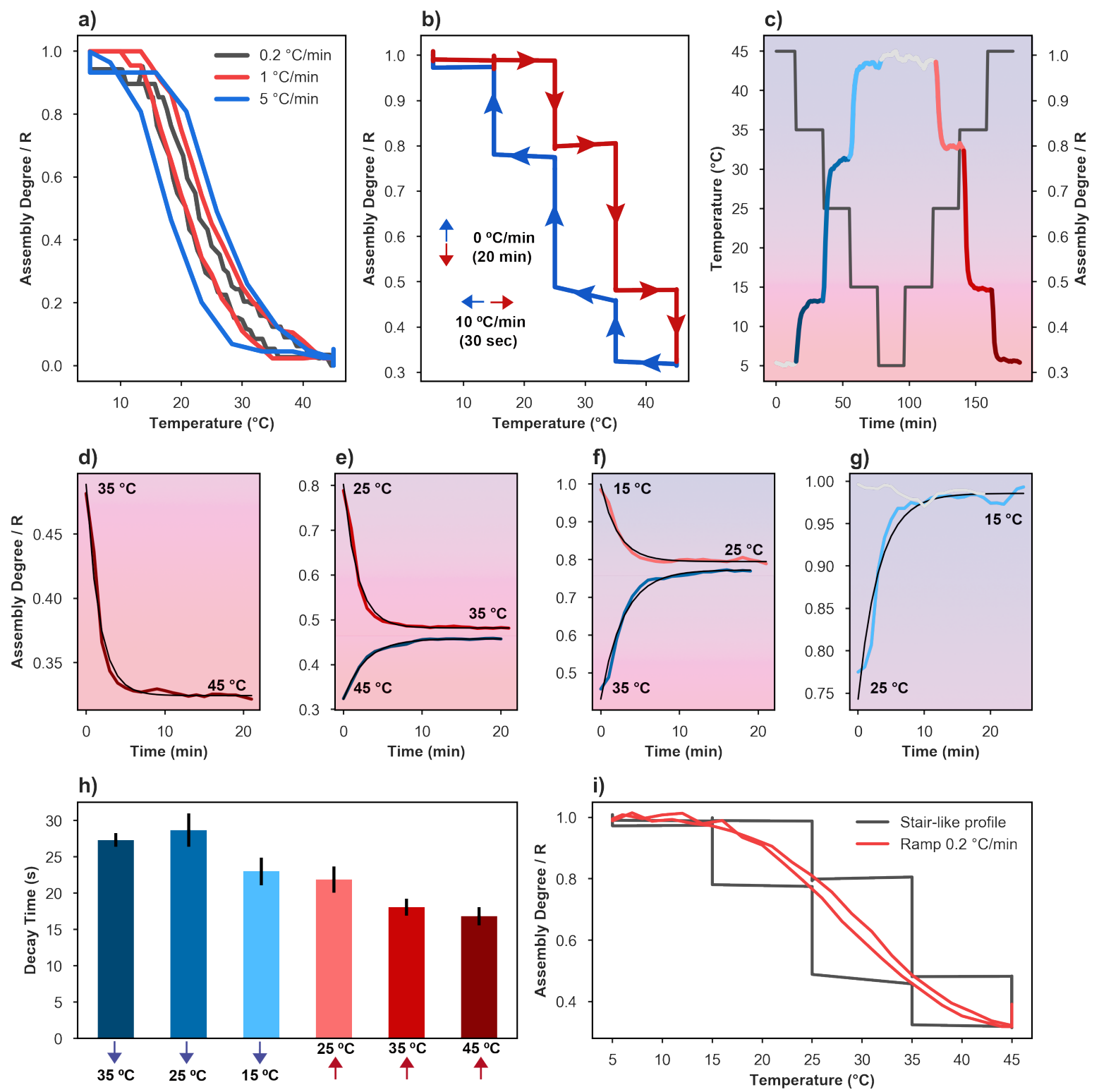

Figure 3: Rate-dependent and rate-independent component of hysteresis. a) The increase of hysteresis width for increasing the velocities of temperature scan, indicating rate-dependent hysteresis. b) The stair-like hysteresis allowing the relaxation (vertical lines) from dispersed and aggregated states. c) Time-dependent profile of relaxation dynamics for all assembly/disassembly processes from b. In grey, temperature profile. (d-g) Relaxation dynamics (taken from c) to given targeted temperatures. The gaps after relaxation to 35 and $25^{\circ} \mathrm{C}$ indicate rate-independent hysteresis. h, i) Decay time constants obtained from $(\mathrm{d}-\mathrm{g})$. The disassembly is faster than clustering. All relaxation curves were fitted by $y=a \exp (-x / b)+c$. i) Assembly degree at continuous hysteresis $\left(0.2^{\circ} \mathrm{C} / \mathrm{min}\right)$ coincide with assembly degree after relaxation in stair-like hysteresis, confirming rate-independent hysteresis. 
seconds more than redispersion (Figure 3h). This difference is an indirect signature of the origin of hysteresis. The clustering that is induced by cooling is a slow process in which the initially free nanoparticles diffuse to encounter each other to form clusters. In terms of the energy landscape, the particles are progressively "trapped" in the energy well. The lack of energy barrier allows the AuNPs to start clustering at the very beginning of the temperature drop. On the contrary, an extra thermal energy is required to induce disassembly making the electrostatic repulsion to overcome vdW attraction. Once this extra energy is supplied, an abrupt redispersion commences, pushing the particles out from the energy well. Here, the extra energy is ensured at each stage of stair-like hysteresis, resulting in faster relaxation profile of heating than cooling. Such reasoning indicates that the assembly temperature is higher than the clustering temperature, hence leading to the emergence of hysteresis.

Both rate-dependent and rate-independent components are sensitive to the nanoparticle size. Increasing the diameter from 12, 19 to $30 \mathrm{~nm}$, the hysteresis broadened for fast and slow velocities of temperature change (Figure 4a,b). The size effect is more pronounced at a fast scan $\left(5{ }^{\circ} \mathrm{C} / \mathrm{min}\right)$, suggesting that the size influences the rate-dependent component. Note that the adjustment of salt concentration was needed to keep the hysteresis centred within the temperature window for different particle diameters. We found that the increase in the diameter by $1 \mathrm{~nm}$ required the decrease of salt concentration by a factor of 0.89 (Figure S9).

To determine the change of rate-independent hysteresis with increasing diameter of nanoparticles we plotted the temperature difference $(\Delta \mathrm{T})$ at the middle height of hysteresis (dashed line in Figure 4a,b) versus the velocity of the temperature scan, as shown in Figure 4c). The intercept of this nearly-linear relation corresponds to the temperature difference at infinitely small velocity of temperature scan, being thus the width of thermodynamic hysteresis. Figure $4 \mathrm{~d}$ confirms that the width increases proportionally with the increase of nanoparticles diameter.

Likewise, the stair-like hysteresis for each nanoparticle size showed that the gaps - the difference of assembly degree $\Delta R$ - increase with diameter of nanoparticles (Figures $4 \mathrm{e}-\mathrm{g}$, 

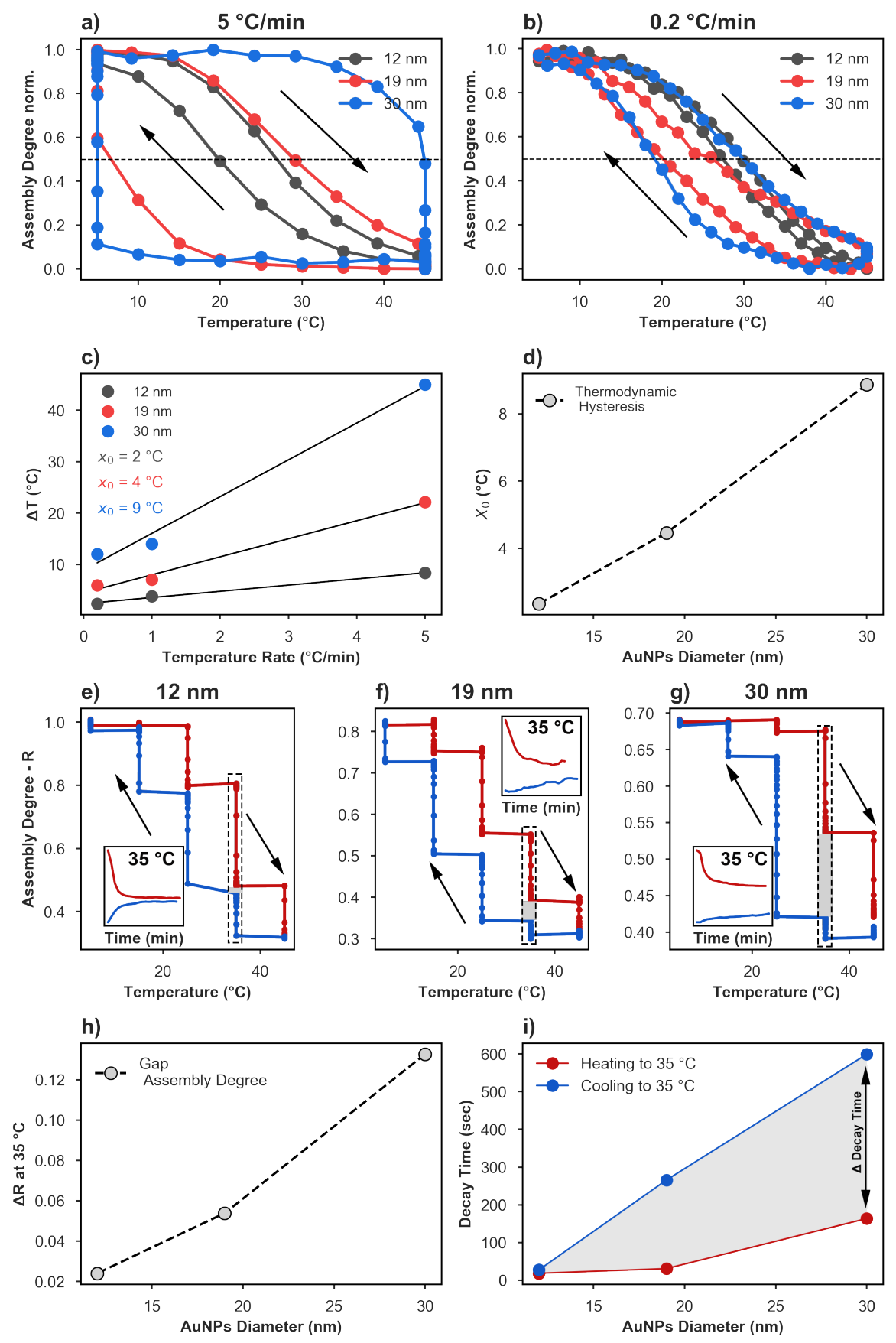

Figure 4: Effect of AuNPs size on rate-dependent and rate independent hysteresis. a-b) Hysteresis loop for 12, 19 and $30 \mathrm{~nm}$ AuNPs at a) $5{ }^{\circ} \mathrm{C} / \mathrm{min}$ and b) $0.2{ }^{\circ} \mathrm{C} / \mathrm{min}$, where arrows indicate the direction of temperature scan. c) Temperature difference $\Delta \mathrm{T}$ at middle height of hysteresis $(R=0.5$, dashed line in a and b) versus velocity of temperature scan. d) The values of intercepts from c $\left(\Delta \mathrm{T}\right.$ at $\left.0{ }^{\circ} \mathrm{C} / \mathrm{min}\right)$. e-f) Stair-like hysteresis for different particle diameter with highlighted gaps at $35{ }^{\circ} \mathrm{C}$ - gray area in dotted rectangle. Insets: relaxation profiles at $35{ }^{\circ} \mathrm{C}$ after heating (red) and cooling (blue). h) Magnitude of gaps, $\Delta R=R_{\text {heating }}-R_{\text {cooling }}$, at $35^{\circ} \mathrm{C}$ for different AuNPs sizes, extracted from plots e,f,g. i) Decay times versus AuNPs diameter for cooling and heating at $35^{\circ} \mathrm{C}$. 
$\mathrm{S} 11, \mathrm{~S} 12)$. It is especially visible for the relaxation at $35^{\circ} \mathrm{C}$ - a grey area in dotted rectangles in Figure $4 \mathrm{c}$-e. The $\Delta R$ follows a nearly linear trend with increasing nanoparticles diameter, indicating that the rate-independent component becomes prominent with increasing the size of nanoparticles (Figure 4h). The analysis of relaxation profiles at $35^{\circ} \mathrm{C}$ shows that the decay times at cooling are higher than decay times at heating as the diameter of nanoparticles increases (Figure 4i). These data indicate that the larger the particles are, the larger the difference between clustering and assembly temperatures, leading to more pronounced rateindependent component of the hysteresis.

\section{Surface potential broadens hysteresis}

Neutralization of surface potential has greater impact on the hysteresis. It is well known, that native surfactants comprising quaternary ammonium ions $\left(\mathrm{CTA}^{+}\right)$and halides counterions (e.g., $\mathrm{Cl}^{-}$) form a stable bilayer on the surface of metallic nanoparticles. ${ }^{33,34}$ Although the surfactant bilayers ensure excellent colloidal stability to the nanoparticles, they hinder the ligand exchange even of thiol-terminated molecules. ${ }^{35-37}$ In the experiments discussed above, we deliberately selected citrate as good leaving ligands to ensure their efficient replacement with phosphine-bearing BSPP. We observed that upon ligand exchange the negative value of $\zeta$-potential is conserved (Figure 5a). This is quite the opposite scenario when replacing a cationic surfactant, such as cetyltrimethylammonium chloride (CTAC), with BSPP, where the sign of $\zeta$-potential is reverted from positive to negative. Since the affinity of BSPP to the gold surface is lower than that of thiolated ligands, it is reasonable to assume that BSPP is unable to replace completely the native CTAC molecules, however, they are capable of reversing the sign of $\zeta$-potential (Figure 5a). Thus, the presence of residual surfactant molecules mixes surface charge, resulting in a more neutral $\zeta$-potential $(-18 \mathrm{mV})$ as compared with $-41 \mathrm{mV}$ of $\mathrm{Au} @ \mathrm{BSPP}$ in which citrate is the starting ligand.

We performed cyclic clustering of BSPP-stabilized nanoparticles $(9 \mathrm{~nm})$ using citrate and $\mathrm{CTAC}$ as the starting ligands, hereafter named as $\mathrm{AuNP}^{-}$and $\mathrm{AuNP}^{ \pm}$, respectively. Figure 

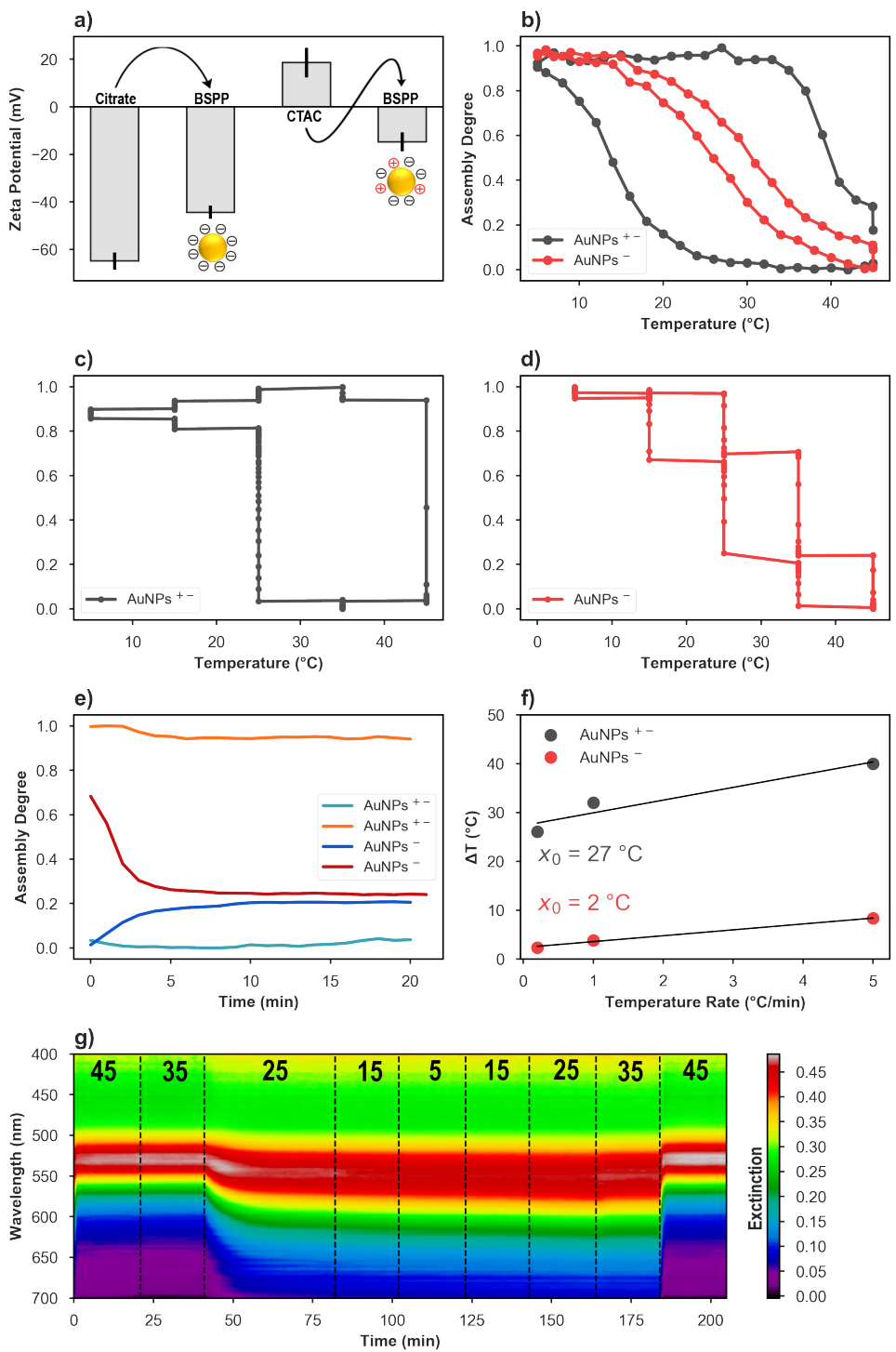

Figure 5: Influence of surface potential of AuNPs on rate-independent hysteresis.a) The difference in surface $\zeta$-potential of nanoparticles before and after ligand exchange using citrate or CTAC as starting stabilizers. b) Hysteresis loops for AuNPs with mixed charges $\left(\mathrm{AuNP}^{ \pm}\right)\left(\mathrm{CTAC}\right.$ as native stabilizer) and AuNPs with negative charges $\left(\mathrm{AuNP}^{-}\right)$(citrate as native stabilizer) at $0.2{ }^{\circ} \mathrm{C} / \mathrm{min}$. c,d) Stair-like hysteresis for $\mathrm{AuNP}^{ \pm}$(c) and for $\mathrm{AuNP}^{-}$(d). e) Change of assembly degree upon relaxation to target temperature of $35^{\circ} \mathrm{C}$ for both types of AuNPs, showing invariance of assembly degree in $\mathrm{AuNP}^{ \pm}$and fast decay at in $\mathrm{AuNP}^{-}$. f) $\Delta \mathrm{T}$ at $R=0.5$ for different temperature rates. The black line visualizes that $\mathrm{AuNP}^{ \pm}$show weak response to an increase of temperature rate, but a highly increased thermodynamic hysteresis for $\mathrm{x}=0{ }^{\circ} \mathrm{C} / \mathrm{min}$. g) Time-dependent spectral changed of AuNP $\mathrm{P}^{ \pm}$from $\mathrm{c}$, showing slow change of assembly degree upon cooling to $25^{\circ} \mathrm{C}$ and burst-like disassembly to $45^{\circ} \mathrm{C}$. Temperature values in upper part. 
5b shows that $\mathrm{AuNP}^{ \pm}$feature much broader hysteresis than $\mathrm{AuNPs}^{-}$at $0.2{ }^{\circ} \mathrm{C} / \mathrm{min}$. At $35^{\circ} \mathrm{C}$, the gap in stair-like hysteresis for $\mathrm{AuNP}^{ \pm}$was much larger than for $\mathrm{AuNP}^{-}$(Figure $5 c, d$, S13). In fact, it remained fully open. Relaxation dynamics to this temperature shows that for $\mathrm{AuNP}^{ \pm}$the assembly degree remains practically invariant over 20 minutes while for $\mathrm{AuNP}^{-}$it relaxed to produce a small gap (Figure 5e). The width of rate-independent hysteresis $\left(\Delta \mathrm{T}\right.$ at $0{ }^{\circ} \mathrm{C} / \mathrm{min}$ ) was estimated to be $\sim 27{ }^{\circ} \mathrm{C}$ for $\mathrm{AuNP}^{ \pm}$(Figure 5f) while for $\mathrm{AuNP}^{-}$it was $\sim 2{ }^{\circ} \mathrm{C}$ (Figure 5f). These results confirm that thermodynamic hysteresis is greatly increased with neutralizing surface potential.

To show that disassembly temperature is higher than clustering temperature we compared the aggregation rate during cooling to $25^{\circ} \mathrm{C}$ and redispersion rate during heating to $45{ }^{\circ} \mathrm{C}$ for $\mathrm{AuNP}^{ \pm}$. The clustering took 50 minutes to complete, redispersion less than a minute (Figure $5 \mathrm{~g}$ ). We speculate that particles with mixes surface charges are less sensitive to temperature change, meaning that larger energy input is required to induce redispersion through electrostatic repulsion, thereby leading to greater hysteresis.

We are also aware of eventual interactions of the nanoparticles with the agarose matrix that can additionally alter the hysteresis. The cationic surfactant molecules can bind to negatively-charged agarose that can additionally increase the temperature difference of clustering and redispersion.

\section{Conclusions}

In summary, we defined experimental parameters to control the hysteretic behaviour in temperature-driven reversible clustering of gold nanoparticles. We showed that ionic strength, controlled by salt and ligand concentrations, modulates the centre of the hysteresis within a temperature range from 5 to $45^{\circ} \mathrm{C}$. The rate of heating or cooling altered its magnitude suggesting that assemblies remain under a kinetic regime (rate-dependent hysteresis). Both the asymmetric relaxation profiles of assembly degree at fixed temperature and the intercept 
of temperature scan velocity at fixed assembly degree defined the value of rate-independent hysteresis for given experimental parameter - diameter of nanoparticles and their surface potential. From a broader perspective, the simplicity of our method for estimation of kinetic and thermodynamics hysteresis can be applied to virtually any chemical system (colloidal, supramolecular) that undergo reversible transition upon the action of a stimulus. Further coupling the hysteresis with chemical transformations can enable switchable catalysis, autocatalytic chemical networks, or nanosystems that store and transmit information.

\section{Associated content}

\section{Supporting Information}

The Supporting Information is available free of charge on the ACS Publications website at DOI:

- TEM and DLS analysis of gold nanoparticles before and after ligand exchange, DLS analysis of temperature-driven clustering of gold nanoparticles in colloidal phase, determination of the size of pores in agarose matrix, effect of agarose concentration on nanoparticles clustering, detailed analysis of hysteresis, effect of temperature scan rate at $1 \mathrm{~mm}$ cuvette, effect of nanoparticles concentration on hysteresis, relaxation behaviour of nanoparticles in $1 \mathrm{~mm}$ cuvette.

\section{Author Information}

\section{Corresponding Author}

Email: marek.grzelczak@dipc.org 


\section{ORCID}

Joscha Kruse: 0000-0001-9123-2437

Stefan Merkens: 0000-0001-5635-8659

Marek Grzelczak: 0000-0002-3458-8450

Andrey Chuvilin: 0000-0002-3712-5638

\section{Notes}

The authors declare no competing financial interest.

\section{Acknowledgement}

This work was supported by the Basque Government (PIBA 2018-34, RIS3 2018222034) and Diputacion Foral de Gipuzkoa (RED2018, RED2019). SM and AC acknowledge support by Spanish MINECO under the Maria de Maeztu Units of Excellence Program (MDM-20160618). S. M. acknowledges funding from the Basque Ministry of Education in the frame of the "Programa Predoctoral de Formación de Personal Investigador no Doctor" (grant reference: PRE-2019-1-0239). Authors thank Jaime Martin and Sara Marina Barbier for their support and access to the optical characterization tool.

\section{References}

(1) Ferrell, J. E.; Machleder, E. M. The biochemical basis of an all-or-none cell fate switch in Xenopus oocytes. Science 1998, 280, 895-898.

(2) Pomerening, J. R.; Sontag, E. D.; Ferrell, J. E. Nat. Cell Biol. 2003, 5, 346-351.

(3) Harfe, B. D.; Scherz, P. J.; Nissim, S.; Tian, H.; McMahon, A. P.; Tabin, C. J. Evidence 
for an expansion-based temporal Shh gradient in specifying vertebrate digit identities. Cell 2004, 118, 517-528.

(4) Das, J.; Ho, M.; Zikherman, J.; Govern, C.; Yang, M.; Weiss, A.; Chakraborty, A. K.; Roose, J. P. Digital signaling and hysteresis characterize ras activation in lymphoid cells. Cell 2009, 136, 337-351.

(5) Morris, K. A. What is Hysteresis? Appl. Mech. Rev. 2012, 64, 00043.

(6) Grzybowski, B. A.; Huck, W. T. S. The nanotechnology of life-inspired systems. Nat. Nanotechnol. 2016, 11, 585-592.

(7) Grzybowski, B. A.; Fitzner, K.; Paczesny, J.; Granick, S. From dynamic self-assembly to networked chemical systems. Chem. Soc. Rev. 2017,

(8) Iida, R.; Mitomo, H.; Matsuo, Y.; Niikura, K.; Ijiro, K. Thermoresponsive Assembly of Gold Nanoparticles Coated with Oligo(Ethylene Glycol) Ligands with an Alkyl Head. J. Phys. Chem. C 2016, 120, 15846-15854.

(9) Grzelczak, M.; Liz-Marzán, L. M.; Klajn, R. Stimuli-responsive self-assembly of nanoparticles. Chem. Soc. Rev. 2019, 48, 1342-1361.

(10) Deng, F.; Wang, Y.; Lu, X.; Ding, T. Probing hidden colloidal transitions with the assistance of surface plasmons. Phys. Chem. Chem. Phys. 2019,

(11) Grötsch, R. K.; Wanzke, C.; Speckbacher, M.; Angı, A.; Rieger, B.; Boekhoven, J. Pathway dependence in the fuel-driven dissipative self-assembly of nanoparticles. $J$. Am. Chem. Soc. 2019, 141, 9872-9878.

(12) Wang, D.; Kowalczyk, B.; Lagzi, I.; Grzybowski, B. A. Bistability and Hysteresis During Aggregation of Charged Nanoparticles. J. Phys. Chem. Lett. 2010, 1, 1459-1462.

(13) Geyer, T.; Born, P.; Kraus, T. Switching between crystallization and amorphous agglomeration of alkyl thiol-coated gold nanoparticles. Phys. Rev. Lett. 2012, 109, 128302. 
(14) Born, P.; Kraus, T. Ligand-dominated temperature dependence of agglomeration kinetics and morphology in alkyl-thiol-coated gold nanoparticles. Phys. Rev. E 2013, 87, 062313.

(15) Nabika, H.; Oikawa, T.; Iwasaki, K.; Murakoshi, K.; Unoura, K. Dynamics of Gold Nanoparticle Assembly and Disassembly Induced by pH Oscillations. J. Phys. Chem. C 2012, 116, 6153-6158.

(16) Tóth-Szeles, E.; Horváth, J.; Holló, G.; Szűcs, R.; Nakanishi, H.; Lagzi, I. Chemically coded time-programmed self-assembly. Mol. Syst. Des. Eng. 2017, 2, 274-282.

(17) Guo, Y.; Ma, Y.; Xu, L.; Li, J.; Yang, W. Conformational Change Induced Reversible Assembly/Disassembly of Poly- 1 -lysine-Functionalized Gold Nanoparticles. J. Phys. Chem. C 2007, 111, 9172-9176.

(18) Liu, F.; Agarwal, S. Thermoresponsive Gold Nanoparticles with Positive UCST-Type Thermoresponsivity. Macromol. Chem. Phys. 2015, 216, 460-465.

(19) Ye, J.; Hou, Y.; Zhang, G.; Wu, C. Temperature-induced aggregation of poly(Nisopropylacrylamide)-stabilized CdS quantum dots in water. Langmuir 2008, 24, 27272731.

(20) Bhattacharjee, R. R.; Chakraborty, M.; Mandal, T. K. Reversible association of thermoresponsive gold nanoparticles: polyelectrolyte effect on the lower critical solution temperature of poly(vinyl methyl ether). J. Phys. Chem. B 2006, 110, 6768-6775.

(21) Kim, D. J.; Kang, S. M.; Kong, B.; Kim, W.-J.; Paik, H.-j.; Choi, H.; Choi, I. S. Formation of Thermoresponsive Gold Nanoparticle/PNIPAAm Hybrids by SurfaceInitiated, Atom Transfer Radical Polymerization in Aqueous Media. Macromol. Chem. Phys. 2005, 206, 1941-1946. 
(22) Li, C.; Wang, C.; Ji, Z.; Jiang, N.; Lin, W.; Li, D. Synthesis of thiol-terminated thermoresponsive polymers and their enhancement effect on optical limiting property of gold nanoparticles. Eur. Polym. J. 2019, 404-410.

(23) Liu, X.-Y.; Cheng, F.; Liu, Y.; Li, W.-G.; Chen, Y.; Pan, H.; Liu, H.-J. Thermoresponsive gold nanoparticles with adjustable lower critical solution temperature as colorimetric sensors for temperature, $\mathrm{pH}$ and salt concentration. J. Mater. Chem. 2010, 20, 278-284.

(24) Hill, A. P.; Kunstmann-Olsen, C.; Grzelczak, M. P.; Brust, M. Entropy-Driven Reversible Agglomeration of Crown Ether Capped Gold Nanoparticles. Chem.: Eur. J. 2018, 24, 3151-3155.

(25) Bishop, K. J. M.; Kowalczyk, B.; Grzybowski, B. A. Precipitation of oppositely charged nanoparticles by dilution and/or temperature increase. J. Phys. Chem. B 2009, 113, $1413-1417$.

(26) Gan, J.; Mei, Z.; Chen, X.; Zhou, Y.; Ge, M.-F. A Modified Duhem Model for RateDependent Hysteresis Behaviors. Micromachines 2019, 10, 680.

(27) He, X.; Aizenberg, M.; Kuksenok, O.; Zarzar, L. D.; Shastri, A.; Balazs, A. C.; Aizenberg, J. Synthetic homeostatic materials with chemo-mechano-chemical self-regulation. Nature 2012, 487, 214-218.

(28) Semenov, S. N.; Kraft, L. J.; Ainla, A.; Zhao, M.; Baghbanzadeh, M.; Campbell, V. E.; Kang, K.; Fox, J. M.; Whitesides, G. M. Autocatalytic, bistable, oscillatory networks of biologically relevant organic reactions. Nature 2016, 537, 656-660.

(29) Klajn, R.; Wesson, P. J.; Bishop, K. J. M.; Grzybowski, B. A. Writing self-erasing images using metastable nanoparticle inks. Angew. Chem. Int. Ed. 2009, 48, 70357039 . 
(30) Liu, Y.; Han, X.; Le He,; Yin, Y. Thermoresponsive assembly of charged gold nanoparticles and their reversible tuning of plasmon coupling. Angew. Chem. Int. Ed. 2012, $51,6373-6377$.

(31) Narayanan, J.; Xiong, J.-Y.; Liu, X.-Y. Determination of agarose gel pore size: Absorbance measurements vis a vis other techniques. J. Phys. Conf. Ser. 2006, 28, 83.

(32) Jones, S. T.; Walsh-Korb, Z.; Barrow, S. J.; Henderson, S. L.; del Barrio, J.; Scherman, O. A. The Importance of Excess Poly(N-isopropylacrylamide) for the Aggregation of Poly(N-isopropylacrylamide)-Coated Gold Nanoparticles. ACS Nano 2016, 10, $3158-3165$.

(33) Nikoobakht, B.; El-Sayed, M. A. Evidence for Bilayer Assembly of Cationic Surfactants on the Surface of Gold Nanorods. Langmuir 2001, 17, 6368-6374.

(34) Gómez-Graña, S.; Hubert, F.; Testard, F.; Guerrero-Martínez, A.; Grillo, I.; LizMarzán, L. M.; Spalla, O. Surfactant (Bi)Layers on Gold Nanorods. Langmuir 2011, 28, 1453-1459.

(35) Dewi, M. R.; Laufersky, G.; Nann, T. A highly efficient ligand exchange reaction on gold nanoparticles: preserving their size, shape and colloidal stability. $R S C$ Advances 2014, 4, 34217-34220.

(36) García, I.; Henriksen-Lacey, M.; Sánchez-Iglesias, A.; Grzelczak, M.; Penadés, S.; LizMarzán, L. M. Residual CTAB Ligands as Mass Spectrometry Labels to Monitor Cellular Uptake of Au Nanorods. J. Phys. Chem. Lett. 2015, 2003-2008.

(37) Zhou, S.; Huo, D.; Goines, S.; Yang, T.-H.; Lyu, Z.; Zhao, M.; Gilroy, K. D.; Wu, Y.; Hood, Z. D.; Xie, M. et al. Enabling Complete Ligand Exchange on the Surface of Gold Nanocrystals through the Deposition and Then Etching of Silver. J. Am. Chem. Soc. 2018, 140, 11898-11901. 